\title{
Qualitative Longitudinal Assessment of Stressors and Coping Mechanisms among Syrian Refugee Families in Turkey
}

\author{
A.Arenliu', S.Weine' ${ }^{2}$, J. Konjufca ${ }^{3}$, N. Bertelsen ${ }^{4}$. \\ 'University o Prishtina- Hasan Prishtina, Psychology, Prishtina, Kosovo. \\ 2University of Illinois, Psychiatry, Chicago, USA. \\ ${ }^{3}$ University of Prishtina- Hasan Prishtina, Psychology, Prishtina, Kosovo. \\ ${ }^{4}$ Koç University, School of Medicine, Istanbul, Turkey.
}

Objectives: This study explores the changes coping mechanisms used by Syrian refugee families in Istanbul to cope with multilevel stressors over 9months. . Rather than focusing on individuals this study focuses on families as unit of analysis. Understanding on how Syrian refugee families cope with displacement and dispossession in a Turkey will contribute to international refugee/migration literature and also pave way to development of family based interventions for refugee populations.

Background: Currently an estimated 68.5 million people are forcibly displaced worldwide of which 25.4 million are refugees and 6.3 million are refugees from the war in Syria (UNHCR, 20l8a). Turkey is presently the world's largest refugee hosting country with about 3.6 million refugees of which are 3.2 million from Syria. Living in Turkey, Syrian refugees cope with multiple daily stressors such as: high housing costs, low wages, exploitation in the labor market, discrimination, and uncertainty about their future (Kaya \& Kiraç, 2016). Despite the increase in school enrollment of refugee children, over $40 \%$ of Syrian refugee children are still not in school (UNICEF, 2018). Available studies with Syrian refugees are predominantly focused on common mental health disorders and are largely based on quantitative methods and report high levels of common mental health problems. This study is informed primarily by Walsh's family resilience framework and Weine's Family Consequences of Refugee Trauma empirical model.

Materials and Methods: We conducted initial and 9-month follow up interviews with 30 Syrian refugee families in Istanbul. Data was coded and analyzed with Atlas/ti.

Results \& Conclusion: The initial assessment identified 22 family stressors in three domains (Surviving war and border crossing; Living in Istanbul; Parenting in Istanbul)

Stressors related to war experience were less pressing compared to concerns over financial issues, schooling of children and assimilation to new culture in second assessment. Seventeen family coping mechanisms were identified initially in four domains (Flexible and Reciprocal Family Organization; Hopeful Family Beliefs and Communication; Staying Connected with Family in Syria and in Exile; Making the Best of Living in a New Country). After 9 months families decreased in connecting with family in Syria and exile compared to coping mechanisms in other domains such as women empowerment, spouse support, hoping for better future for children, be optimistic and feeling at home in Turkey. Over time, stressors related to the war in Syria diminished whereas displacement stressors increased. These changes over time in stressors and coping strategies can help inform family focused interventions that address current family stressors by strengthening current family coping strategies. Table below provides some characteristics of families getting better and still struggling. These changes over time in stressors and coping strategies can help inform family focused interventions that address current family stressors by strengthening current family coping strategies.

Families getting better

-Children learning Turkish

-Children adjusting to school

-Family reunification in

Istanbul

-Improved self-reported mental health status of some with CMD

-Families adjusting to life in Ist. -Some families moved to better apartments -Some families were able to visit Syria for Bayram -Some families are benefiting from CBO's

-Families keep open communication as much as possible
Families still struggling

-Most families had some children not in school -Many families had some children working -Working condition for majority remain poor -No improvement in family finances -Lack of accessible language learning opportunities for adults that work -Sense of being exhausted -Uncertainty regarding situation in Syria. 